\title{
Association between Helicobacter pylori VacA antigens and gastric cancer depends on the detection method used: immunoblot versus neutralization of the vacuolating activity of $\mathrm{VacA}$
}

\author{
Correspondence \\ Lizbeth López-Carrillo \\ lizbeth@insp.mx
}

Received 2 August 2007

Accepted 20 September 2007

\author{
Guadalupe Ayala, Julia Torres-Mena and Lizbeth López-Carrillo
} National Institute of Public Health of Mexico. Av. Universidad No. 655, Col. Santa Ma. Ahuacatitlán,
62508 Cuernavaca, Morelos, Mexico

\begin{abstract}
There are inconsistent findings on the association between Helicobacter pylori anti-VacA antibodies and gastric cancer (GC) risk. The lack of optimally sensitive and specific methods to detect anti-VacA antibodies may partly be responsible for this discrepancy. The aim of this study was to compare the association between GC and the presence of anti-VacA antibodies using two different detection methods. A secondary analysis of sera collected in a hospital-based case-control study in three geographical areas of Mexico was performed. Anti-VacA antibodies were determined by a neutralization assay and an immunoblot assay in serum samples of 203 histologically confirmed GC cases and 430 age- and sex-matched controls. $H$. pylori lgG status was determined by immunoblotting and $H$. pylori CagA status data was available for this study. Unconditional logistic regression models were used to estimate the association between anti-VacA antibodies and GC by histological type (diffuse and intestinal). Anti-VacA seroprevalence was higher using neutralization compared with immunoblotting: 68.5 vs $44.3 \%$ for cases and 60.5 vs $44.2 \%$ for controls. A significant association between anti-VacA antibodies and diffuse $\mathrm{GC}$ was found using neutralization [odds ratio $(\mathrm{OR})=1.69,95 \% \mathrm{Cl} 1.08-2.66$ ], but the association did not remain significant after adjusting for CagA status (OR 1.37, $95 \% \mathrm{Cl}$ $0.81-2.32)$. No association between anti-VacA antibodies and GC was found when using immunoblotting. Thus, the association between anti-VacA antibodies and GC partly depends on the detection method used. These results do not support an independent role for VacA in GC risk in the presence of CagA seropositivity and strengthen the importance of CagA as a potential risk factor for GC.
\end{abstract}

\section{INTRODUCTION}

Less than $3 \%$ of subjects infected with Helicobacter pylori develop gastric cancer (GC) (Peek \& Blaser, 2002). Several virulence factors produced by $H$. pylori are now thought to be implicated in the carcinogenesis process (Xia \& Talley, 2001; Hocker \& Hohenberger, 2003). Of the bacterial factors associated with GC, the most extensively studied are probably the cytotoxin CagA (Hatakeyama, 2003) and the vacuolating cytotoxin VacA (de Figueiredo Soares et al., 1998). However, other virulence factors also have activities that could induce cell transformation by affecting cell metabolism and/or causing tissue damage in general. Among these are the BabA2 adhesin, whose presence is associated with the existence of pre-neoplastic gastric lesions ( $\mathrm{Yu}$ et al., 2002), and a urease, an enzyme that

Abbreviations: GC, gastric cancer; OR, odds ratio; SVA, supernatants with vacuolating activity. releases ammonia, which has been suggested to be the cause of an acceleration of cytokine-induced apoptosis in gastric epithelial cells (Igarashi et al., 2001). A novel apoptosis-inducing protein encoded by the $H$. pylori HP1118 gene has been isolated (Shibayama et al., 2003), and recently it was demonstrated that the co-chaperonin GrosES is implicated in the induction of pro-inflammatory cytokine and neoplastic changes (Lin et al., 2006).

The H. pylori VacA cytotoxin is a virulence factor potentially linked to GC. VacA causes cell vacuolation in cell culture, produces significant epithelial cell damage and increases the level of apoptosis in epithelial cells (Galmiche et al., 2000; Kuck et al., 2001; Cover et al., 2003); this in turn enhances the hyperproliferative response, altering the balance of gastric homeostasis, which changes gastric epithelial cell turnover and permits the persistence of mutated cells. Studies suggest that these processes may promote carcinogenesis (Galmiche et al., 2000; Xia \& Talley, 2001). 
The clinical and epidemiological evidence regarding the relationship between VacA and GC is unclear (de Figueiredo Soares et al., 1998; Shimoyama et al., 1999; Yamaoka et al., 1999; Enroth et al., 2000; Figueroa et al., 2002). Among patients with GC, some clinical studies have reported positive correlations between antibody host response to VacA and the presence of VacA-positive $H$. pylori strains (de Figueiredo Soares et al., 1998; GarzaGonzalez et al., 2004). Some epidemiological studies have shown an increased risk of GC in seropositive VacA subjects (Enroth et al., 2000; Figueroa et al., 2002), but not all studies have reported these findings (Shimoyama et al., 1999; Yamaoka et al., 1999). Two possible explanations for the inconsistent reporting of the VacA/GC association are: (i) the lack of control for key confounding factors such as H. pylori CagA status, and (ii) significant differences in the sensitivity and specificity of the immunoblot kits used to determine the $H$. pylori VacA status; among these are: Helicoblot (HB2.0 or HB2.1; Genelabs Diagnostics) (de Figueiredo Soares et al., 1998; Enroth et al., 2000; GarzaGonzalez et al., 2004), the Chiron RIBA Recombinant Immunoblot assay (Chiron) (Shimoyama et al., 1999; Yamaoka et al., 1999) and other homemade immunoblot assays (Hirai et al., 1994; Rudi et al., 1997; Figueroa et al., 2002).

Here, we compared the association between GC and the presence of anti-VacA antibodies using two different detection methods; in addition, we adjusted for CagA status. The study was conducted with serum samples from Mexico, where approximately $80 \%$ of the adult population are seropositive for $H$. pylori (Torres et al., 1998) and where GC is still a leading cause of death by cancer (Secretaría de Salud, 1993). Unlike the rest of the world (Parkin et al., 1997), the incidence of this type of cancer is not decreasing.

\section{METHODS}

This study undertook a secondary analysis of serum samples obtained between 1994 and 1996 in a hospital-based case-control study that was conducted in three distinct geographical areas of Mexico. The areas were selected based on the variability of $H$. pylori seroprevalence and dietary patterns. More detailed information about the methodology of this study has been published elsewhere (López-Carrillo et al., 2003).

Cases. Inclusion criteria for case subjects were: histologically confirmed adenocarcinoma of the stomach, either intestinal or diffuse type; no previous history of any other cancer; at least 20 years of age; and resident in the study area for at least the previous 6 months. Eligible cases were recruited from 13 tertiary, public, social security hospitals in Mexico, distributed as follows: seven from Mexico City (Federal District), three from the city of Puebla (State of Puebla) and three from the city of Merida (State of Yucatan). A single expert cancer pathologist determined the histological variety of the tumour (intestinal or diffuse), following the criteria established by Lauren (1965). Information about the anatomical subsite location of the tumour was not available for this study. In total, 281 patients who met all of the eligibility criteria were identified (approx. $75 \%$ of the total number of GC patients reported to the Mexican National Cancer Registry during the study period of recruitment for the study areas) and 261 agreed to enrol in the study, giving a participation rate of $92.9 \%$.

Controls. For each case subject, we recruited a minimum of two age-, sex- and residence-matched control subjects. Controls were randomly identified in selected departments of the participating hospitals. Eligibility criteria included: no previous diagnosis of malignant tumour, diet-related disease (gastritis, peptic ulcer, liver cirrhosis or diabetes mellitus) or immunosuppressive condition; same age $( \pm 5$ years) as the index case; and resident in the same study area as the index case for at least the previous 6 months. The most common diagnoses among controls were: circulatory system diseases (other than hypertension) $(18.8 \%)$; diseases of the nervous system and the sensory organs (except psychiatric disorders) (16.1\%); osteomuscular and connective tissue disorders $(14.4 \%)$; injuries and poisonings $(10.9 \%)$; and respiratory diseases $(10.0 \%)$. Other less common diagnoses were genitourinary diseases and skin problems. We also recruited control subjects who were healthy companions of patients and those attending the hospitals for preventive care purposes, such as vaccination. The participation rate for the control group was $94.6 \%$ (523 out of 553 eligible subjects).

Interviews. After informed consent had been obtained from each subject, they were questioned about their socioeconomic, clinical and dietary characteristics. The interviews were performed in the participating hospitals by trained personnel.

Serum samples. Blood samples $(10 \mathrm{ml})$ were drawn from each subject. After centrifugation, serum aliquots were obtained and stored at $-70{ }^{\circ} \mathrm{C}$ until the samples were tested. Serum samples from a total of 203 cases and 430 controls were available for this study.

The H. pylori status of each subject was determined. H. pylori IgG antibodies were determined by immunoblot as described below. The H. pylori CagA status was available for this study and the ELISA procedures used to determine it have been described elsewhere (Lopez-Carrillo et al., 2004).

Production of cytotoxin and assessment of vacuolating activity. A high level toxin-producing $H$. pylori strain $(7494, \mathrm{~s} 1 / \mathrm{m} 1$ genotype and $\operatorname{cag} A^{+}$) was used as the source for vacuolating cytotoxin preparation. Colonies obtained from one blood agar plate [tryptic soy agar (Difco) supplemented with $5 \%$ sheep blood plus $10 \mu \mathrm{g}$ vancomycin $\mathrm{ml}^{-1}$ ] were inoculated into $100 \mathrm{ml}$ sulfite-free Brucella broth containing $0.2 \%$ cyclodextrin (Sigma) and cultured as described previously (Ayala et al., 2006). The cultures were centrifuged at $15000 \mathrm{~g}$ for $20 \mathrm{~min}$ at $4{ }^{\circ} \mathrm{C}$. The supernatants were then pooled and this fraction was termed 'supernatants with vacuolating activity' (SVA). Analysis by $10 \%$ SDS-PAGE showed that this fraction was composed of proteins of different molecular mass, among them a band corresponding to VacA $(\sim 87 \mathrm{kDa})$. The SVA fraction was used as the source of VacA for the neutralization assays, as well as antigen for immunoblot analysis. Determination of vacuolating activity for the SVA fraction was conducted essentially as described by Cover et al. (1990). The assay was carried out by the addition of a fixed amount of total SVA protein $(6.1 \mu \mathrm{g})$ to confluent HeLa cells. To estimate quantitatively the vacuolating activity of the SVA fraction, a neutral red uptake assay was used as reported elsewhere (de Bernard et al., 1998) and is described below. The protein concentration of the SVA was determined using the Lowry method (Lowry et al., 1951).

H. pylori anti-VacA antibody status was determined by two methods: a VacA-neutralizing antibody assay and an in-house immunoblot assay.

Neutralization of VacA cytotoxin activity. The serum from each patient was inactivated as described elsewhere (Cover et al., 1992) and 
then diluted with Dulbecco's modified Eagle's medium (DMEM) cellculture medium. Inhibition of VacA activity using patient sera was performed according to a method reported elsewhere (Cover et al., 1992) and modified as follows: equal volumes of $H$. pylori SVA containing $6.1 \mu \mathrm{g}$ total protein and serum from each subject were pre-incubated for $1 \mathrm{~h}$ at $37{ }^{\circ} \mathrm{C}$. The starting amount of protein in each serum sample was $9.5 \mu \mathrm{g}$ and ten further twofold dilutions from $1: 4$ to $1: 2048$, were carried out in DMEM culture medium in 96-well plates. Next, $100 \mu \mathrm{l}$ from each well containing the SVA/serum mixture was transferred to a second 96-well plate containing confluent HeLa cells $\left(7 \times 10^{3}\right.$ cells per well $)$. The plates were incubated for $18 \mathrm{~h}$ at $37{ }^{\circ} \mathrm{C}$ with $5 \% \mathrm{CO}_{2}$. For each serum sample, two controls were included: (i) HeLa cells with SVA but without serum (positive control), and (ii) HeLa cells with DMEM culture medium alone (negative control). Experiments were carried out in duplicate. Both vacuolization and inhibition were evaluated by neutral red uptake assay (de Bernard et al., 1998). Cells were stained for $8 \mathrm{~min}$ with $0.05 \%$ neutral red. After washing the cells twice with $0.3 \%$ BSA in PBS, intravacuolar neutral red was extracted with $70 \%$ ethanol and $0.37 \% \mathrm{HCl} . A_{540}$ was determined using a Multiskan EX ELISA reader (Thermo Electron). All assays were performed in duplicate. Results were expressed as the mean $A_{540}$ of experimental wells, minus background $A_{540}$ (the mean $A_{540}$ of wells containing HeLa cells incubated with medium only). The net $A_{540}$ value was used to calculate the cut-off value.

The cut-off point was determined by comparing the net $A_{540}$ value of wells containing both the SVA fraction and the serum being tested. The net $A_{540}$ values obtained for each of ten serial dilutions (described above) were compared by analysis of variance. The positive/negative cut-off point was determined using the lowest dilution for which a statistically significant difference was observed in the mean net $A_{540}$ between two consecutive dilutions. The cut-off point was set as 0.16 absorbance units at a dilution of $1: 32$. Thus, a serum was judged to be positive (i.e. showed neutralizing activity) if the $A_{540}$ value was lower than 0.16 at a dilution of $1: 32$. Sera with $A_{540} \geqslant 0.16$ were considered negative (i.e. no neutralizing activity).

H. pylori and VacA cytotoxin immunoblot assay. The SVA fraction was concentrated approximately 50 -fold by ultrafiltration with a $10 \mathrm{kDa}$ cut-off (Millipore). Proteins were separated by $10 \%$ SDS-PAGE and transferred to nitrocellulose membranes (Millipore). The filter was blocked for $1 \mathrm{~h}$ with buffer containing $10 \%(\mathrm{v} / \mathrm{v})$ skimmed milk in $20 \mathrm{mM}$ Tris/ $\mathrm{HCl}(\mathrm{pH} 7.5)$ containing $0.5 \mathrm{M} \mathrm{NaCl}$. The membranes were cut into strips and incubated overnight at room temperature with serum samples from each patient at a $1: 200$ dilution. The strips were washed and further incubated for $1 \mathrm{~h}$ at room temperature with alkaline phosphatase-conjugated goat antihuman IgG (Sigma). A serum sample was considered to be $H$. pyloripositive if three or more immunoreactive bands were present on the strip; otherwise, the sample was considered negative. The sample was also judged to be VacA-positive if a band with a molecular mass of $\sim 87 \mathrm{kDa}$ was recognized. To confirm that the $87 \mathrm{kDa}$ band corresponded to VacA, one strip was incubated with anti-VacA antibody (bN-20; Santa Cruz Biotechnology). Colour reactions were developed with 5-bromo-4-chloro-3-indolylphosphate and nitro blue tetrazolium.

Statistical analyses. The general characteristics of cases and controls (age, sex, etc.), as well as H. pylori status (IgG, CagA and anti-VacA antibodies), were compared using Student's $t$ test and $\chi^{2}$ statistics. Crude and adjusted risks (by age and CagA status) for GC according to the presence of $H$. pylori IgG antibodies and VacA status were estimated using unconditional logistic regression models for all adenocarcinomas and then stratified for histological type of GC (i.e. intestinal and diffuse).

\section{RESULTS AND DISCUSSION}

The general characteristics of the study population are presented in Table 1. Age, sex and residence distribution were similar, and no significant difference was found between cases and controls regarding socioeconomic level. Intestinal GC was more frequent than diffuse GC in this population.

\section{H. pylori status and anti-VacA and anti-CagA seroprevalence}

There was a significantly higher proportion of cases positive for $H$. pylori IgG compared with the controls (91.1 vs $83.2 \%$; Table 2). A statistically significant difference in CagA-positive status was also found between cases and controls (78.6 vs $66.0 \%$ ).

In this study, VacA seroprevalence was analysed by two methods: neutralization of vacuolating activity of VacA cytotoxin in vitro and immunoblot assay. VacA seroprevalence was higher by neutralization, compared with immunoblotting: 68.5 vs $44.3 \%$ for cases and 60.5 vs $44.2 \%$ for controls (Table 2). A borderline significant difference was found between cases and controls for the presence of anti-VacA antibodies (68.5 vs $60.5 \%$ ) when these were determined by neutralization. In contrast, when immunoblotting was used to detect anti-VacA antibodies, lower and similar percentages were found in both cases and controls (44.3 and $44.2 \%$, respectively). The discrepancy between the two methods may be due to the fact that most

Table 1. General characteristics of the study population

\begin{tabular}{|c|c|c|c|}
\hline Characteristic & $\begin{array}{c}\text { Cases }(n=203) \\
(\%)\end{array}$ & $\begin{array}{c}\text { Controls } \\
(n=430)(\%)\end{array}$ & $P$ value $^{x}$ \\
\hline Age (years) & & & 0.883 \\
\hline$<40$ & 10.8 & 10.0 & \\
\hline $40-50$ & 18.7 & 18.1 & \\
\hline $50-60$ & 21.2 & 24.2 & \\
\hline $60-70$ & 29.1 & 30.0 & \\
\hline$>70$ & 20.2 & 17.7 & \\
\hline Sex & & & 0.803 \\
\hline Male & 43.8 & 42.8 & \\
\hline Female & 56.2 & 57.2 & \\
\hline Residence & & & 0.328 \\
\hline Mexico City & 30.5 & 28.6 & \\
\hline Puebla State & 43.9 & 40.0 & \\
\hline Yucatan State & 25.6 & 31.4 & \\
\hline \multicolumn{3}{|c|}{ Change in socioeconomic level $\dagger$} & 0.560 \\
\hline Same or less & 50.5 & 48.0 & \\
\hline Improvement & 49.5 & 52.0 & \\
\hline \multicolumn{4}{|l|}{ Histological type } \\
\hline Intestinal & 37.2 & - & - \\
\hline Diffuse & 62.8 & - & - \\
\hline
\end{tabular}

${ }^{\star} P$ value from $\chi^{2}$ test.

$\dagger$ Current level compared with socioeconomic level in childhood. 
Table 2. $H$. pylori status (including virulence factors) in the study population

\begin{tabular}{|lccc|}
\hline Positive for: & $\begin{array}{c}\text { Cases } \\
(\boldsymbol{n}=\mathbf{2 0 3})(\boldsymbol{\%})\end{array}$ & $\begin{array}{c}\text { Controls } \\
(\boldsymbol{n}=\mathbf{4 3 0})(\boldsymbol{\%})\end{array}$ & $\boldsymbol{P}$ value $^{\star}$ \\
\hline IgG (Western blot) & 91.1 & 83.2 & 0.008 \\
VacA (immunoblot) & 44.3 & 44.2 & 0.984 \\
VacA (neutralization assay) & 68.5 & 60.5 & 0.051 \\
CagA (ELISA) & 78.6 & 66.0 & 0.002 \\
\hline
\end{tabular}

${ }^{\star} P$ value from $\chi^{2}$ test.

anti-VacA antibodies present in $H$. pylori-infected patients recognize conformational epitopes of $\mathrm{VacA}$, whilst linear epitopes of VacA are recognized to a lesser degree (Figueiredo et al., 2001). This weak reactivity of VacA in immunoblots using sera from patients with active $H$. pylori infection has been reported previously (Lepper et al., 2004). The failure to recognize linear VacA epitopes could potentially result in differences in sensitivity and specificity between immunoblotting and neutralization. Therefore, detection of anti-VacA antibodies by inhibition of VacA activity in vitro should produce more reliable data than immunoblot assays. It is important to note that human serum from $H$. pylori-infected patients, as well as from uninfected persons, contains non-specific VacA-neutralizing activity that does not depend on anti-VacA IgG (Cover et al., 1992). However, such an effect would not have affected our results at the dilution used $(1: 32)$, as the amount of serum protein at the positive/negative cut-off point was very low $(\sim 0.30 \mu \mathrm{g})$, so any non-specific neutralizing activity would be negligible.

\section{Association of virulence factor with GC}

Table 3 shows that although immunoblotting and neutralization both detected anti-VacA antibodies, only when the neutralization assay was used were we able to find a significant odds ratio (OR 1.69, $95 \%$ CI 1.08-2.66). A significant association between $H$. pylori IgG antigens and GC risk was also detected (OR 2.37, 95\% CI 1.15-4.89) (data not shown).

It is well accepted that individuals infected with $H$. pylori strains expressing both CagA and VacA are at higher risk of developing GC (Rudi et al., 1997; Enroth et al., 2000; Figueroa et al., 2002). However, there is still little information to show the extent to which the presence of both virulence factors contributes to the development of GC. Studies of VacA biological activity (Galmiche et al., 2000; Kuck et al., 2001; Cover et al., 2003; Karita et al., 2005) suggest that it plays an indirect role in the development of GC, mainly by inducing apoptosis, which results in the atrophy associated with the carcinogenic process. Although our results showed a significant association between anti-VacA antibodies and diffuse GC when detected by neutralizing antibodies, the association did not remain significant after adjusting for anti-CagA antibodies (OR 1.37, $95 \%$ CI 0.81-2.32). Thus, our results strengthen the importance of $\mathrm{CagA}$ as a potential risk factor for GC, despite the fact that its presence improves the assessment of GC risk for VacA.

VacA and CagA are involved in different steps of the pathogenic process. VacA plays an essential role in the initial colonization of $H$. pylori and the subsequent epithelial cell damage of the stomach mucosa (Salama et al., 2001). VacA also suppresses the local immune response, which promotes the presence of a chronic, persistent $H$. pylori infection (Gebert et al., 2004). CagApositive $H$. pylori strains possess an intact Cag pathogenicity island, which is associated with activation of the inflammatory response and mucosal immunity, as well as with altered regulation of multiple genes associated with proliferation and differentiation of gastric epithelial cells. The function of the CagA protein itself is not yet clear, but recent reports have associated it with transformation of the gastric epithelium. This might indicate that the development of GC would only occur if the H. pylori infection is chronic and if the infecting strain is CagA-positive (Amieva et al., 2003; Hatakeyama, 2004). Chronic inflammatory

Table 3. ORs for the effect of $H$. pylori VacA on the risk of intestinal and diffuse GC

\begin{tabular}{|c|c|c|c|c|c|c|}
\hline \multirow[t]{2}{*}{ VacA status } & \multicolumn{3}{|c|}{ Intestinal GC } & \multicolumn{3}{|c|}{ Diffuse GC } \\
\hline & Cases/controls $(n)^{\star}$ & OR $(95 \% \mathrm{CI}) \dagger$ & OR $(95 \% \mathrm{CI}) \ddagger$ & Cases/controls $(n)^{\star}$ & OR $(95 \% \mathrm{CI}) \dagger$ & OR $(95 \% \mathrm{CI}) \ddagger$ \\
\hline \multicolumn{7}{|l|}{ Immunoblot } \\
\hline Negative & $36 / 239$ & 1.0 & 1.0 & $69 / 239$ & 1.0 & 1.0 \\
\hline Positive & $32 / 191$ & $1.11(0.66-1.86)$ & $0.89(0.52-1.55)$ & $46 / 191$ & $0.83(0.55-1.27)$ & $0.68(0.43-1.06)$ \\
\hline \multicolumn{7}{|l|}{$\begin{array}{l}\text { Neutralization } \\
\text { assay }\end{array}$} \\
\hline Negative & $27 / 170$ & 1.0 & 1.0 & $32 / 170$ & 1.0 & 1.0 \\
\hline Positive & $41 / 260$ & $0.99(0.59-1.67)$ & $0.71(0.39-1.30)$ & $83 / 260$ & $1.69(1.08-2.66)$ & $1.37(0.81-2.32)$ \\
\hline
\end{tabular}

${ }^{\star}$ Cell numbers that do not add up to the total sample size are due to missing values.

$\dagger$ Crude OR.

¥OR adjusted by age and CagA status. 
changes are linked to cancer, thus explaining the relative importance of CagA versus VacA in GC. However, we cannot exclude the participation of the co-chaperonin GroES of $H$. pylori in the induction of inflammation and neoplastic changes (Lin et al., 2006), reflecting the multiple pathogenic possibilities displayed by this bacterium.

VacA is a highly expressed protein in vivo (Boonjakuakul et al., 2004) and some studies have shown that carriers of $H$. pylori strains that efficiently produce VacA are at higher risk of GC. However, few studies have evaluated the relationship of GC with the presence of anti-VacA antibodies (Rudi et al., 1997; de Figueiredo Soares et al., 1998; Enroth et al., 2000; Figueroa et al., 2002) and results have been inconsistent (Shimoyama et al., 1999; Yamaoka et al., 1999). Thus, the anti-VacA/GC association is questionable, and earlier controversial results may partially be explained by their different methods and the criteria of the assay.

This study showed that the association between anti-VacA antibodies and GC risk depends not only on the type of serological test used to detect anti-VacA antibodies and CagA, but also on the CagA status. This last situation does not support an independent role for VacA in GC risk.

\section{ACKNOWLEDGEMENTS}

This work was supported by grants $37195 \mathrm{M}$ from CONACYT and FOSISS-06-ST-165. The authors thank Dr Javier Torres for analytical determination of the serum CagA status. We extend our special thanks to Geny Fierros-Zárate for valuable help.

\section{REFERENCES}

Amieva, M. R., Vogelmann, R., Covacci, A., Tompkins, L. S., Nelson, W. J. \& Falkow, S. (2003). Disruption of the epithelial apicaljunctional complex by Helicobacter pylori CagA. Science $\mathbf{3 0 0}$, 1430-1434.

Ayala, G., Torres, L., Espinosa, M., Fierros-Zarate, G., Maldonado, V. \& Melendez-Zajgla, J. (2006). External membrane vesicles from Helicobacter pylori induce apoptosis in gastric epithelial cells. FEMS Microbiol Lett 260, 178-185.

Boonjakuakul, J. K., Syvanen, M., Suryaprasad, A., Bowlus, C. L. \& Solnick, J. V. (2004). Transcription profile of Helicobacter pylori in the human stomach reflects its physiology in vivo. J Infect Dis $\mathbf{1 9 0}$, 946-956.

Cover, T. L., Dooley, C. P. \& Blaser, M. J. (1990). Characterization of and human serologic response to proteins in Helicobacter pylori broth culture supernatants with vacuolizing cytotoxin activity. Infect Immun 58, 603-610.

Cover, T. L., Cao, P., Murthy, U. K., Sipple, M. S. \& Blaser, M. J. (1992). Serum neutralizing antibody response to the vacuolating cytotoxin of Helicobacter pylori. J Clin Invest 90, 913-918.

Cover, T. L., Krishna, U. S., Israel, D. A. \& Peek, R. M., Jr (2003). Induction of gastric epithelial cell apoptosis by Helicobacter pylori vacuolating cytotoxin. Cancer Res 63, 951-957.

de Bernard, M., Moschioni, M., Papini, E., Telford, J., Rappuoli, R. \& Montecucco, C. (1998). Cell vacuolization induced by Helicobacter pylori VacA toxin: cell line sensitivity and quantitative estimation. Toxicol Lett 99, 109-115. de Figueiredo Soares, T., de Magalhães Queiroz, D. M., Mendes, E. N., Rocha, G. A., Rocha Oliveira, A. M., Alvares Cabral, M. M. \& de Oliveira, C. A. (1998). The interrelationship between Helicobacter pylori vacuolating cytotoxin and gastric carcinoma. Am J Gastroenterol 93, 1841-1847.

Enroth, H., Wolfgang, K., Engstrand, L., Nyrén, O. \& Rohan, T. (2000). Helicobacter pylori strain types and risk of gastric cancer: a casecontrol study. Cancer Epidemiol Biomarkers Prev 9, 981-985.

Figueiredo, C., Quint, W. \& Nouhan, N. (2001). Assessment of Helicobacter pylori vacA and cagA genotypes and host serological response. J Clin Microbiol 39, 1339-1344.

Figueroa, G., Troncoso, M., Toledo, M. S., Faundez, G. \& Acuna, R. (2002). Prevalence of serum antibodies to Helicobacter pylori VacA and CagA and gastric diseases in Chile. J Med Microbiol 51, 300-304.

Galmiche, A., Rassow, J., Doye, A., Cagnol, S., Chambard, J. C., Contamin, S., de Thillot, V., Just, l., Ricci, V. \& other authors (2000). The N-terminal $34 \mathrm{kDa}$ fragment of Helicobacter pylori vacuolating cytotoxin targets mitochondria and induces cytochrome $c$ release. EMBO J 19, 6361-6370.

Garza-Gonzalez, E., Bosques-Padilla, F. J., Perez-Perez, G. I., Flores-Gutierrez, J. P. \& Tijerina-Menchaca, R. (2004). Association of gastric cancer, HLA-DQA1, and infection with Helicobacter pylori $\mathrm{CagA}^{+}$and $\mathrm{VacA}^{+}$in a Mexican population. J Gastroenterol 39, $1138-1142$.

Gebert, B., Fischer, W. \& Haas, R. (2004). The Helicobacter pylori vacuolating cytotoxin: from cellular vacuolation to immunosuppressive activities. Rev Physiol Biochem Pharmacol 152, 205-220.

Hatakeyama, M. (2003). Helicobacter pylori CagA - a potential bacterial oncoprotein that functionally mimics the mammalian Gab family of adaptor proteins. Microbes Infect 5, 143-150.

Hatakeyama, M. (2004). Oncogenic mechanisms of the Helicobacter pylori CagA protein. Nat Rev Cancer 4, 688-694.

Hirai, M., Azuma, T., Ito, S., Kato, T., Kohli, Y. \& Fuliki, N. (1994). High prevalence of neutralizing activity to Helicobacter pylori cytotoxin in serum of gastric-carcinoma patients. Int J Cancer 56, 56-60.

Hocker, M. \& Hohenberger, P. (2003). Helicobacter pylori virulence factors - one part of a big picture. Lancet 362, 1231-1233.

Igarashi, M., Kitada, Y., Yoshiyama, H., Takagi, A., Miwa, T. \& Koga, Y. (2001). Ammonia as an accelerator of tumor necrosis factor $\alpha$-induced apoptosis of gastric epithelial cells in Helicobacter pylori infection. Infect Immun 69, 816-821.

Karita, M., Teramukai, S., Matsumoto, S. \& Shibuta, H. (2005). Intracellular VacA is a valuable marker to predict whether Helicobacter pylori induces progressive atrophic gastritis that is associated with the development of gastric cancer. Dig Dis Sci 50, 56-64.

Kuck, D., Kolmerer, B., Iking-Konert, C., Krammer, P. H., Stremmel, W. \& Rudi, J. (2001). Vacuolating cytotoxin of Helicobacter pylori induces apoptosis in the human gastric epithelial cell line AGS. Infect Immun 69, 5080-5087.

Lauren, P. (1965). The two histological main types of gastric carcinoma: diffuse and so-called intestinal-type carcinoma. An attempt at a histo-clinical classification. Acta Pathol Microbiol Scand 64, 31-49.

Lepper, P. M., Moricke, A., Vogt, K., Bode, G. \& Trautmann, M. (2004). Comparison of different criteria for interpretation of immunoglobulin $G$ immunoblotting results for diagnosis of Helicobacter pylori infection. Clin Diagn Lab Immunol 11, 569-576.

Lin , Y. F., Wu, M. S., Chang, C. C., Lin, S. W., Lin, J. T., Sun, Y. J., Chen, D. S. \& Chow, L. P. (2006). Comparative immunoproteomics of identification and characterization of virulence factors from 
Helicobacter pylori related to gastric cancer. Mol Cell Proteomics 5, 1484-1496.

López-Carrillo, L., Lopez-Cervantes, M., Robles-Diaz, G., RamirezEspitia, A., Mohar-Betancourt, A., Meneses-Garcia, A., Lopez-Vidal, Y. \& Blair, A. (2003). Capsaicin consumption, Helicobacter pylori positivity and gastric cancer in Mexico. Int J Cancer 106, 277-282.

López-Carrillo, L., Torres-Lopez, J., Galvan-Portillo, M., Munoz, L. \& Lopez-Cervantes, M. (2004). Helicobacter pylori-CagA seropositivity and nitrite and ascorbic acid food intake as predictors for gastric cancer. Eur J Cancer 40, 1752-1759.

Lowry, O. H., Rosebrough, N. J., Farr, A. L. \& Randall, R. J. (1951). Protein measurement with the Folin phenol reagent. J Biol Chem 193, 265-275.

Parkin, D. M., Whelan, S. L., Ferlay, J., Raymond, L. \& Young, J. (1997). Cancer Incidence in Five Continents, vol. VII. IARC Scientific Publication No. 143. Lyon: IARC Press.

Peek, R. M., Jr \& Blaser, M. J. (2002). Helicobacter pylori and gastrointestinal tract adenocarcinoma. Nat Rev Cancer 2, 28-37.

Rudi, J., Kolb, C., Maiwald, M., Zuna, I., von Herbay, A., Galle, P. R. \& Stremmel, W. (1997). Serum antibodies against Helicobacter pylori proteins VacA and CagA are associated with increased risk for gastric adenocarcinoma. Dig Dis Sci 42, 1652-1659.

Salama, N. R., Otto, G., Tompkins, L. \& Falkow, S. (2001). Vacuolating cytotoxin of Helicobacter pylori plays a role during colonization in a mouse model of infection. Infect Immun 69, 730-736.
Secretaría de Salud (1993). Compendio de Estadísticas de Morbilidad por Neoplasias Malignas 1982-1989. Mexico City: Registro Histopatológico de Neoplasias Malignas (in Spanish).

Shibayama, K., Kamachi, K., Nagata, N., Yagi, T., Nada, T., Doi, Y., Shibata, N., Yokoyama, K., Yamane, K. \& other authors (2003). A novel apoptosis-inducing protein from Helicobacter pylori. Mol Microbiol 47, 443-451.

Shimoyama, T., Neelam, B., Fukuda, S., Tanaka, M., Munakata, A. \& Crabtree, J. E. (1999). VacA seropositivity is not associated with the development of gastric cancer in a Japanese population. Eur $J$ Gastroenterol Hepatol 11, 887-890.

Torres, J., Leal-Herrera, Y., Perez-Perez, G., Gomez, A., CamorlingaPonce, M., Cedillo-Rivera, R., Tapia-Conyer, R. \& Muñoz, O. (1998). A community-based seroepidemiologic study of Helicobacter pylori infection in Mexico. J Infect Dis 178, 1089-1094.

Xia, H. H. \& Talley, N. J. (2001). Apoptosis in gastric epithelium induced by Helicobacter pylori infection: implications in gastric carcinogenesis. Am J Gastroenterol 96, 16-26.

Yamaoka, Y., Kodama, T., Kashima, K. \& Graham, D. Y. (1999). Antibody against Helicobacter pylori CagA and VacA and the risk for gastric cancer. J Clin Pathol 52, 215-218.

Yu, J., Leung, W. K., Go, M. Y., Chan, M. C., To, K. F., Ng, E. K., Chan, F. K., Ling, T. K., Chung, S. C. \& Sung, J. J. (2002). Relationship between Helicobacter pylori babA2 status with gastric epithelial cell turnover and premalignant gastric lesions. Gut 51, 480-484. 